\title{
On Some Open Problems in Monotonic and Conservative Learning
}

\author{
Sanjay Jain ${ }^{a, 1}$ \\ ${ }^{a}$ School of Computing, National University of Singapore, Singapore 117417. Email: \\ sanjay@comp.nus.edu.sg
}

\begin{abstract}
In this paper we solve some open problems in monotonic and conservative learning.
\end{abstract}

Key words: Theory of Computation, Inductive Inference, Monotonicity, Conservativeness.

\section{Introduction}

Consider the following learning situation. A learner receives data about the environment, one piece at a time. As the learner is receiving the data, it conjectures its hypothesis about how the environment works. The learner may be considered to be successful if the sequence of hypotheses it conjectures stabilizes to a correct explanation of the environment. This is essentially the paradigm of inductive inference introduced by Gold [Gol67].

Formal definitions of the concepts informally introduced in the Introduction are given in Section 2. In this paper, we will be focusing on language learning. In this situation, the environment takes the form of a language from some class of languages. The data might be a text for the language (that is, it contains all and only the elements of the language, given one element at a time, in arbitrary order with repetition allowed), or may be an informant for the language (that is, it contains membership information about every element $x$ : for each $x$, the data informs the learner about whether $x$ belongs to the language or not). The hypothesis of the learner takes the form of indices (codes) of languages. These indices are interpreted in some hypothesis space (index $i$ will represent the $i$-th language in the hypothesis space). We will always take the learners to

$\overline{1}$ Supported in part by NUS grant number R252-000-308-112. 
be algorithmic and hypothesis space to be a recursively enumerable sequence of (either recursive or r.e.) languages. The hypothesis space might be class preserving (that is, the hypothesis space consists of only the languages in the class being learned), or the hypothesis space may be class comprising (that is, the hypothesis space may consist of other languages, besides the languages in the class being learned) [ZL95].

A learner is strongly monotonic [Jan91] if its later conjectures contain its earlier conjectures: that is the languages represented by the sequence of hypotheses conjectured grow monotonically. A learner is iterative [LZ96,WC80,Wie76], if its new conjecture depends only on the latest datum and its previous conjecture.

One often considers classes of languages that are indexed families [Ang80]: that is, membership question for languages in the class can be effectively decided from the index of the language. We refer the reader to [LZ96] for a recent survey on learning indexed families. Lange and Zeugmann [LZ92] asked whether there exists an indexed family which can be strongly monotonically identified by a learner from informant, but which cannot be strongly monotonically identified by an iterative learner from informant. We show that there exists an indexed family which can be strongly monotonically identified using a class preserving hypothesis space but cannot be strongly monotonically identified by an iterative learner, even if the iterative learner is free in the choice of the hypothesis space used. This contrasts with the situation for learning from texts, where every indexed family which can be strongly monotonically identified can also be strongly monotonically identified by an iterative learner using some appropriate hypothesis space [LZ92].

A conservative learner does not change its conjecture unless the data contradict its hypothesis [Ang80]. Lange and Zeugmann [LZ93] asked whether there exists an indexed family which can be learned from texts using only a bounded number of mind changes, but cannot be conservatively learned from texts. In this paper we answer this question positively by showing that there exists an indexed family which can be learned from texts using at most two mind changes, but which cannot be conservatively learned. Here note that [LZ93] showed that every indexed family which can be learned using at most one mind change can be conservatively learned.

\section{Preliminaries}

Any unexplained recursion theoretic notation is from [Rog67]. $N$ denotes the set of natural numbers. $\min (S)$ denotes the minimum element of the set $S$. $\operatorname{card}(S)$ denotes the cardinality of the set $S$. A language is a subset of $N$. 
We let $L$, with or without subscripts, superscripts and the like, range over languages. $\emptyset$ denotes the empty set (language). We let $\mathcal{L}$ range over sets of languages. $\mathcal{L}$ is said to be an indexed family iff there exists a recursive function $f$ and a listing $L_{0}, L_{1}, \ldots$ of languages in $\mathcal{L}$ such that $\mathcal{L}=\left\{L_{i}: i \in N\right\}$, and $f(i, x)=1$ iff $x \in L_{i}$.

Let $\langle\cdot, \cdot\rangle$ denote a fixed computable bijective mapping from $N \times N$ to $N$.

We let $W_{0}, W_{1}, \ldots$ denote a fixed standard acceptable numbering of all the recursively enumerable sets (languages). $\mathcal{E}$ denotes the set of all recursively enumerable languages.

A text $T$ is a mapping from $N$ into $N \cup\{\#\}$. A sequence $\sigma$ is an initial segment of a text. SEQ denotes the set of all finite sequences. The content of a text $T$, denoted content $(T)$, is the set of natural numbers in the range of $T$. content $(\sigma)$ for a sequence $\sigma$ is defined similarly. $T$ is a text for $L$ iff $\operatorname{content}(T)=L$. Intuitively, a text $T$ for $L$ represents the presentation of elements of $L$ to the learner, with \#'s representing pauses in the presentation (for example, the only text for $\emptyset$ is $\#^{\infty}$ ).

An informant $I$ is a mapping from $N$ into $(N \times\{0,1\})$ such that for all $x$, exactly one of $(x, 1)$ and $(x, 0)$ is in the range of $I$. An information segment is an initial segment of an informant. SEG denotes the set of all information segments. PosInfo $(I)$ denotes the set $\{x:(x, 1)$ is in the range of $I\}$. $\operatorname{NegInfo}(I)$ denotes the set $\{x:(x, 0)$ is in the range of $I\}$. PosInfo $(\sigma)$ and $\operatorname{NegInfo}(\sigma)$ are defined similarly for information segment $\sigma$. $I$ is an informant for $L$ iff $\operatorname{PosInfo}(I)=L$ and $\operatorname{NegInfo}(I)=N-L$.

$\Lambda$ denotes the empty sequence/information segment. $|\sigma|$ denotes the length of sequence/information segment $\sigma . T[n]$ (respectively, $I[n]$ ) denotes the initial segment of text $T$ (respectively, informant $I$ ) with length $n$.

$\sigma \diamond \tau$ denotes the concatenation of $\sigma$ and $\tau$. We sometimes abuse notation and use $\sigma \diamond x$ (or $\sigma \diamond(x, b)$, for $b \in\{0,1\}$ ) to denote the concatenation of sequence $\sigma$ with a sequence containing just the element $x$ (just the pair $(x, b)$ ).

A learning machine is an algorithmic mapping (maybe partial) from SEQ (or SEG) to $N \cup\{$ ? $\}$. Intuitively, ? denotes that the learner does not wish to make a conjecture on the current data. This is useful when the number of mind changes allowed to the learner is limited. Intuitively, $\mathbf{M}(T[0]), \mathbf{M}(T[1]), \mathbf{M}(T[2]), \ldots$ can be considered as the sequence of conjectures made by $\mathbf{M}$ on $T$. A learner $\mathbf{M}$ converges on $T$ to $i$ (written: $\mathbf{M}(T)=i$ ), iff for all but finitely many $n$, $\mathbf{M}(T[n])=i$. Convergence on informants is defined similarly.

The conjectures of the learning machine are interpreted in some hypothesis space $\mathcal{H}=H_{0}, H_{1}, \ldots$ Thus a conjecture $i$ of the learner $\mathbf{M}$ is interpreted 
as the learner conjecturing the language $H_{i}$. In this paper, a hypothesis space $\mathcal{H}=H_{0}, H_{1}, \ldots$ is always an r.e. family of languages, that is, $\left\{\langle i, x\rangle: x \in H_{i}\right\}$ is recursively enumerable. In some cases (for the positive sides of our results), we even take the hypothesis space to be an indexed family: that is $\left\{\langle i, x\rangle: x \in H_{i}\right\}$ is recursive.

Definition 1 [Gol67] Suppose $\mathcal{H}=H_{0}, H_{1}, \ldots$ is a hypothesis space.

(a.1) M TxtEx-identifies $L$ (using hypothesis space $\mathcal{H}$ ) iff for all texts $T$ for $L$, (i) for all $n \in N, \mathbf{M}(T[n]) \downarrow$ and (ii) there exists an $i$ such that $\mathbf{M}(T)=i$ and $H_{i}=L$.

(a.2) M TxtEx-identifies $\mathcal{L}$ (using hypothesis space $\mathcal{H}$ ) iff $\mathbf{M}$ TxtEx-identifies each $L \in \mathcal{L}$.

(b.1) M InfEx-identifies $L$ (using hypothesis space $\mathcal{H}$ ) iff for all informants $I$ for $L$, (i) for all $n \in N, \mathbf{M}(I[n]) \downarrow$ and (ii) there exists an $i$ such that $\mathbf{M}(I)=i$ and $H_{i}=L$.

(b.2) $\mathbf{M}$ InfEx-identifies $\mathcal{L}$ (using hypothesis space $\mathcal{H}$ ) iff $\mathbf{M}$ InfEx-identifies each $L \in \mathcal{L}$.

We will use the terms identify and learn as synonyms.

Definition 2 [Jan91] Suppose $\mathbf{M}$ is a learner from informants, and $\mathcal{H}=$ $H_{0}, H_{1}, \ldots$ is the hypothesis space used by $\mathbf{M}$.

$\mathbf{M}$ is said to be strongly monotonic on $\mathcal{L}$ iff for all $L \in \mathcal{L}$, for all informants $I$ for $L$, for all $m, n$ with $m<n$ such that $\mathbf{M}(I[m])$ and $\mathbf{M}(I[n])$ are not?, $H_{\mathbf{M}(I[m])} \subseteq H_{\mathbf{M}(I[n])}$.

$\mathbf{M}$ is said to InfSMon-identify $\mathcal{L}$ (using hypothesis space $\mathcal{H}$ ) iff $\mathbf{M}$ is strongly monotonic on $\mathcal{L}$ and $\mathbf{M}$ InfEx-identifies $\mathcal{L}$.

Definition 3 [LZ96,WC80,Wie76] Suppose M is a learner from informants. $\mathbf{M}$ is said to be iterative iff for all $\sigma, \tau \in \mathrm{SEG}$ and $(w, b) \in(N \times\{0,1\})$, $\mathbf{M}(\sigma)=\mathbf{M}(\tau)$ implies $\mathbf{M}(\sigma \diamond(w, b))=\mathbf{M}(\tau \diamond(w, b))$.

One can similarly define strongly monotonic and iterative learners for learning from texts (though we will not be using them for this paper).

Note that for TxtEx and InfEx-learning (along with strongly monotonic and conservative versions of these criteria) one may assume without loss of generality that the learner is total. However, for iterative learning one cannot assume so. That is the reason we explicitly required that the learner is defined on all initial segments of texts for languages in the class $\mathcal{L}$ being learned.

Definition 4 [Ang80] Suppose $\mathbf{M}$ is a learner from texts and $\mathcal{H}=H_{0}, H_{1}, \ldots$ 
is the hypothesis space used by $\mathbf{M}$.

$\mathbf{M}$ is said to be conservative on $\mathcal{L}$ iff for all $L \in \mathcal{L}$, for all texts $T$ for $L$, for all $m, n$ with $m<n$ such that $\mathbf{M}(T[m]) \neq$ ?, if content $(T[n]) \subseteq H_{\mathbf{M}(T[m])}$, then $\mathbf{M}(T[m])=\mathbf{M}(T[n])$.

$\mathbf{M}$ is said to conservatively identify $\mathcal{L}$ (using $\mathcal{H}$ as hypothesis space) iff $\mathbf{M}$ is conservative on $\mathcal{L}$ and $\mathbf{M}$ TxtEx-identifies $\mathcal{L}$ (using $\mathcal{H}$ as hypothesis space).

\section{Results}

The following solves an open problem from [LZ92].

Theorem 5 There exists an indexed family $\mathcal{L}$ such that

(a) some learner InfSMon-identifies $\mathcal{L}$ (using a class preserving hypothesis space $\mathcal{H}=H_{0}, H_{1}, \ldots$, where one can effectively decide membership question for $\left.H_{i}\right)$;

(b) no iterative learner can InfSMon-identify $\mathcal{L}$ (using any hypothesis space).

Proof. Let $X_{0}=\{\langle 0,0\rangle\}$.

For $y>0$, let $X_{y}=X_{0} \cup\{\langle 1, y\rangle\} \cup\{\langle y+1, z\rangle: z \in N\}$.

For $y>0$ and any finite subset $D$ of $N$, let $R_{y}^{D}=X_{0} \cup\{\langle 1, y\rangle,\langle 1, y+1\rangle\} \cup$ $\{\langle y+1, z\rangle: z \in D\}$.

Let $\mathcal{L}=\left\{X_{y}: y \in N\right\} \cup\left\{R_{y}^{D}: y>0, D\right.$ is a finite subset of $\left.N\right\}$.

It is easy to verify that $\mathcal{L}$ is an indexed family.

The following learner $\mathbf{M}$ InfSMon-identifies $\mathcal{L}$. For hypothesis space we assume a $1-1$ indexing of $\mathcal{L}$ where one can effectively, from any $y \in N$ and any finite set $D$, find an index for $X_{y}$ and $R_{y+1}^{D}$ (note that such an indexing can easily be constructed). For ease of notation, we just indicate the hypothesis of the learner as "index for $L$ " rather than going into details of indexing.

For $\tau \in \mathrm{SEG}, \mathbf{M}(\tau)$ is defined as follows.

If $\langle 1, y\rangle \notin \operatorname{PosInfo}(\tau)$ for any $y$, then $\mathbf{M}(\tau)=$ index for $X_{0}$;

Else, if there exists a $y>0$ such that $\langle 1, y\rangle \in \operatorname{PosInfo}(\tau)$, and $\langle 1, y-1\rangle,\langle 1, y+$ $1\rangle \in \operatorname{NegInfo}(\tau)$, then $\mathbf{M}(\tau)=$ index for $X_{y}$ (note that, for informants for languages in the class, there can be at most one such $y$ ); 
Else, if $\operatorname{PosInfo}(\tau)=R_{y}^{D}$ for some $y>0$ and finite $D$, then $\mathbf{M}(\tau)=$ index for $R_{y}^{D}$

Otherwise the learner repeats its previous hypothesis (i.e., $\mathbf{M}(\tau)=\mathbf{M}(\sigma)$, where $\tau=\sigma \diamond(w, b)$, for some $(w, b) \in(N \times\{0,1\}))$.

It is easy to verify that the learner M InfSMon-identifies $\mathcal{L}$.

We now show that no iterative learner can InfSMon-identify $\mathcal{L}$ using any hypothesis space. Suppose by way of contradiction that iterative learner $\mathbf{M}$ InfSMon-identifies $\mathcal{L}$ using hypothesis space $\mathcal{H}=H_{0}, H_{1}, \ldots$

Let $\sigma$ be a stabilizing sequence for $\mathbf{M}$ on input $X_{0}$ (for learning from informants) [Ful90,BB75]. That is, let $\sigma$ be such that (a) $\operatorname{PosInfo}(\sigma) \subseteq X_{0}$, (b) $\operatorname{NegInfo}(\sigma) \subseteq N-X_{0}$, (c) for all $\tau$ such that $\operatorname{PosInfo}(\tau) \subseteq X_{0}$ and $\operatorname{NegInfo}(\tau) \subseteq N-X_{0}, \mathbf{M}(\sigma \diamond \tau)=\mathbf{M}(\sigma)$. Note that there exists such a stabilizing sequence as $\mathbf{M}$ InfEx identifies $\mathcal{L}$ (see [BB75]). Let $y>0$ be such that $(\{\langle 1, y\rangle,\langle 1, y+1\rangle\} \cup\{\langle y+1, z\rangle: z \in N\}) \cap(\operatorname{PosInfo}(\sigma) \cup \operatorname{NegInfo}(\sigma))=\emptyset$. Let $I$ be an informant for $X_{y}$, except that it does not contain information about $\langle 1, y+1\rangle$ (that is, $\left.\operatorname{PosInfo}(I)=X_{y}, \operatorname{NegInfo}(I)=\left(N-X_{y}\right)-\{\langle 1, y+1\rangle\}\right)$. Now $\mathbf{M}$ on input $\sigma \diamond I$ must converge to an index for $X_{y}$ (since $\mathbf{M}$ converges on $\sigma \diamond(\langle 1, y+1\rangle, 0) \diamond I$ to an index for $X_{y}, \mathbf{M}$ is iterative and $\mathbf{M}(\sigma)=$ $\mathbf{M}(\sigma \diamond(\langle 1, y+1\rangle, 0)))$.

Let $\tau$ be an initial sequence of $\sigma \diamond I$ such that $\mathbf{M}(\tau)$ is an index for $X_{y}$. Then, $\mathbf{M}$ fails to strongly monotonically identify the set $\operatorname{PosInfo}(\tau) \cup\{\langle 1, y+$ $1\rangle,\langle 1, y\rangle,\langle 0,0\rangle\}$ (which belongs to $\mathcal{L}$ ) from an informant for it which extends $\tau$ (since $\left.H_{\mathbf{M}(\tau)}=X_{y} \nsubseteq(\operatorname{PosInfo}(\tau) \cup\{\langle 1, y+1\rangle,\langle 1, y\rangle,\langle 0,0\rangle\})\right)$. A contradiction.

This contrasts with the situation for learning from texts, where every indexed family which can be strongly monotonically identified from texts can be strongly monotonically identified by an iterative learner from texts (using some appropriate hypothesis space) [LZ92].

The following solves an open problem mentioned in [LZ93]. Here note that [LZ93] showed that every indexed family which can be learned using at most one mind change can be conservatively learned. So the mind change result obtained below is optimal.

Theorem 6 There exists an indexed family $\mathcal{L}$ such that

(a) some learner $\mathbf{M} \mathbf{T x t E x}$-identifies $\mathcal{L}$ using at most two mind changes on any input text (that is, for all texts $T, \operatorname{card}(\{n: ? \neq \mathbf{M}(T[n]) \neq \mathbf{M}(T[n+$ $1])\}) \leq 2$ ) using a class preserving hypothesis space $\mathcal{H}=H_{0}, H_{1}, \ldots$, where 
one can effectively decide membership question for $H_{i}$;

(b) $\mathcal{L}$ cannot be conservatively TxtEx-identified by any learner using the standard acceptable numbering $W_{0}, W_{1}, \ldots$ as hypothesis space (and thus using any recursively enumerable hypothesis space).

Proof. Let $\mathbf{M}_{0}, \mathbf{M}_{1}, \ldots$ denote a recursive enumeration of all learning machines.

Let $X_{i}=\{\langle i, x\rangle: x \in N\}$.

Let $T_{i}(j)=\langle i, j\rangle$. Thus $T_{i}$ is a text for $X_{i}$.

Let $S_{i}=\left\{\langle s, r\rangle: s \geq 1\right.$ and one can verify within $r$ steps that $\mathbf{M}_{i}\left(T_{i}[s]\right) \downarrow$ and $\left.\operatorname{content}\left(T_{i}[s]\right) \subset W_{\mathbf{M}_{i}\left(T_{i}[s]\right)}\right\}$.

Here $\subset$ denotes proper subset. Note that $S_{i}$ is a recursive set, a decision procedure for which can be found effectively from $i$.

If $S_{i}$ is not empty, then let $\left\langle s_{i}, r_{i}\right\rangle=\min \left(S_{i}\right)$.

Let $\mathcal{L}=\left\{X_{i}: i \in N\right\} \cup\left\{\operatorname{content}\left(T_{i}\left[s_{i}\right]\right): S_{i} \neq \emptyset\right\}$.

Note that $\mathcal{L}$ is an indexed family. To see this, let $L_{2 j}=X_{j}$, and $L_{2 j+1}=$ content $\left(T_{i_{j}}\left[s_{i_{j}}\right]\right)$, where $i_{0}, i_{1}, \ldots$ is a $1-1$ recursive enumeration of the set $\{w$ : $\left.S_{w} \neq \emptyset\right\}$. Then, $\mathcal{L}=\left\{L_{j}: j \in N\right\}$. Note that $L_{0}, L_{1}, \ldots$ is a $1-1$ numbering for $\mathcal{L}$ and for all $i \geq 0$, one can effectively obtain a $j$ such that $L_{j}=X_{i}$, and for $i$ such that $S_{i} \neq \emptyset$, one can effectively find a $j$ such that $L_{j}=\operatorname{content}\left(T_{i}\left[s_{i}\right]\right)$.

Furthermore, $\mathcal{L}$ cannot be conservatively identified: if $S_{i}=\emptyset$, then $\mathbf{M}_{i}$ does not TxtEx-identify $X_{i}$; if $S_{i} \neq \emptyset$, then $\mathbf{M}_{i}$ on $T_{i}\left[s_{i}\right]$ outputs a proper superset of content $\left(T_{i}\left[s_{i}\right]\right)$, and thus $\mathbf{M}_{i}$ cannot conservatively identify content $\left(T_{i}\left[s_{i}\right]\right)$.

On the other hand the following learner $\mathbf{M}$ TxtEx-identifies $\mathcal{L}$ using at most two mind changes. For this, we use the hypothesis space $H_{j}=L_{j}$. For ease of notation, we just indicate the hypothesis of the learner as "index for $L$ ".

If content $(\sigma)=\emptyset$, then $\mathbf{M}(\sigma)=$ ?;

Else, if there does not exist an $i$ such that $\emptyset \subset \operatorname{content}(\sigma) \subset X_{i}$, then, $\mathbf{M}(\sigma)$ repeats its previous hypothesis (that is, $\mathbf{M}(\sigma)=\mathbf{M}\left(\sigma^{\prime}\right)$, where $\sigma=\sigma^{\prime} \diamond w$, for some $w \in N \cup\{\#\})$;

Else, let $i$ be such that $\emptyset \subset \operatorname{content}(\sigma) \subset X_{i}$. If it can be verified within $|\sigma|$ steps that $S_{i} \neq \emptyset$ and content $(\sigma) \subseteq \operatorname{content}\left(T_{i}\left[s_{i}\right]\right)$, then $\mathbf{M}(\sigma)$ is the index for content $\left(T_{i}\left[s_{i}\right]\right)$; otherwise $\mathbf{M}(\sigma)$ is the index for $X_{i}$. 
It is easy to verify that the above learner TxtEx-identifies $\mathcal{L}$. Also, it makes at most two mind changes on any input text (possibly from the index for $X_{i}$ to the index for content $\left(T_{i}\left[s_{i}\right]\right)$, and then to the index for $\left.X_{i}\right)$.

\section{Acknowledgements}

We would like to thank Thomas Zeugmann for helpful discussions and comments on the problems and the paper. We also thank the anonymous referees for several helpful comments.

\section{References}

[Ang80] D. Angluin. Inductive inference of formal languages from positive data. Information and Control, 45:117-135, 1980.

[BB75] L. Blum and M. Blum. Toward a mathematical theory of inductive inference. Information and Control, 28:125-155, 1975.

[Ful90] M. Fulk. Prudence and other conditions on formal language learning. Information and Computation, 85:1-11, 1990.

[Gol67] E. M. Gold. Language identification in the limit. Information and Control, 10:447-474, 1967.

[Jan91] K. P. Jantke. Monotonic and non-monotonic inductive inference. New Generation Computing, 8:349-360, 1991.

[LZ92] S. Lange and T. Zeugmann. Types of monotonic language learning and their characterization. In Proceedings of the Fifth Annual Workshop on Computational Learning Theory, pages 377-390. ACM Press, 1992.

[LZ93] S. Lange and T. Zeugmann. Language learning with bounded number of mind changes. In Proceedings of the Tenth Annual Symposium on Theoretical Aspects of Computer Science, pages 682-691. Springer-Verlag, 1993. Lecture Notes Computer Science, 665.

[LZ96] S. Lange and T. Zeugmann. Incremental learning from positive data. Journal of Computer and System Sciences, 53:88-103, 1996.

[LZ96] S. Lange and T. Zeugmann and S. Zilles. Learning indexed families of recursive languages from positive data: A survey. Theoretical Computer Science, 397:194-232, 2008.

[Rog67] H. Rogers. Theory of Recursive Functions and Effective Computability. McGraw-Hill, 1967. Reprinted by MIT Press in 1987. 
[WC80] K. Wexler and P. Culicover. Formal Principles of Language Acquisition. MIT Press, 1980.

[Wie76] R. Wiehagen. Limes-Erkennung rekursiver Funktionen durch spezielle Strategien. Journal of Information Processing and Cybernetics (EIK), 12:93-99, 1976.

[ZL95] T. Zeugmann and S. Lange. A guided tour across the boundaries of learning recursive languages. In K. P. Jantke and S. Lange, editors, Algorithmic Learning for Knowledge-Based Systems, volume 961 of Lecture Notes in Artificial Intelligence, pages 190-258. Springer-Verlag, 1995. 